\title{
The MicroBooNE Experiment and the Low-Energy Excess
}

\author{
Wouter Van De Pontseele, ${ }^{1,2}$ on behalf of the MicroBooNE collaboration \\ ${ }^{1}$ University of Oxford, Oxford OX1 2JD, United Kingdom \\ ${ }^{2}$ Harvard University, Cambridge, Massachusetts 02138, USA
}

\begin{abstract}
MicroBooNE is the first phase of Fermilab's Short Baseline Neutrino (SBN) Liquid Argon Time Projection Chamber (LArTPC) programme. It aims to improve the understanding of the observed excess of lowenergy electromagnetic events at MiniBooNE. The current status of the low-energy excess search in MicroBooNE is presented.
\end{abstract}

Keywords: MicroBooNE, Electron neutrino, LArTPC, Low-Energy Excess DOI: 10.31526/ACP.NDM-2020.32

\section{THE LOW ENERGY EXCESS}

Neutrino oscillations between the three active neutrino flavours are well established. These oscillations are unable to explain a set of anomalies observed in the past decades characterised by $L / E_{v} \approx 1 \mathrm{meV}^{-1}$, where, $L$ denotes the distance between the neutrino source and the detector, $E_{v}$ is the neutrino energy. These anomalies could point in the direction of an additional sterile neutrino. An overview of the status of the anomalies is given in [1]. In the context of MicroBooNE two specific results employing an acceleratorbased muon neutrino source are especially relevant. In the beginning of the century, the LSND experiment observed a an excess of electron anti-neutrino events which has been interpreted as a possible $\bar{v}_{u} \rightarrow \bar{v}_{e}$ appearance signal [2]. MiniBooNE, a mineral oil Cherenkov detector was build at a similar $L / E_{v}$ to verify the observed anomaly. fig. 1 illustrates the result, containing an excess of low-energy electromagnetic events. This excess can be interpreted as an additional oscillation or by background mis-modelling. Due to its detector technology, MiniBooNE is unable to differentiate between electron and photon showers, crucial to identify the origin of the anomaly.
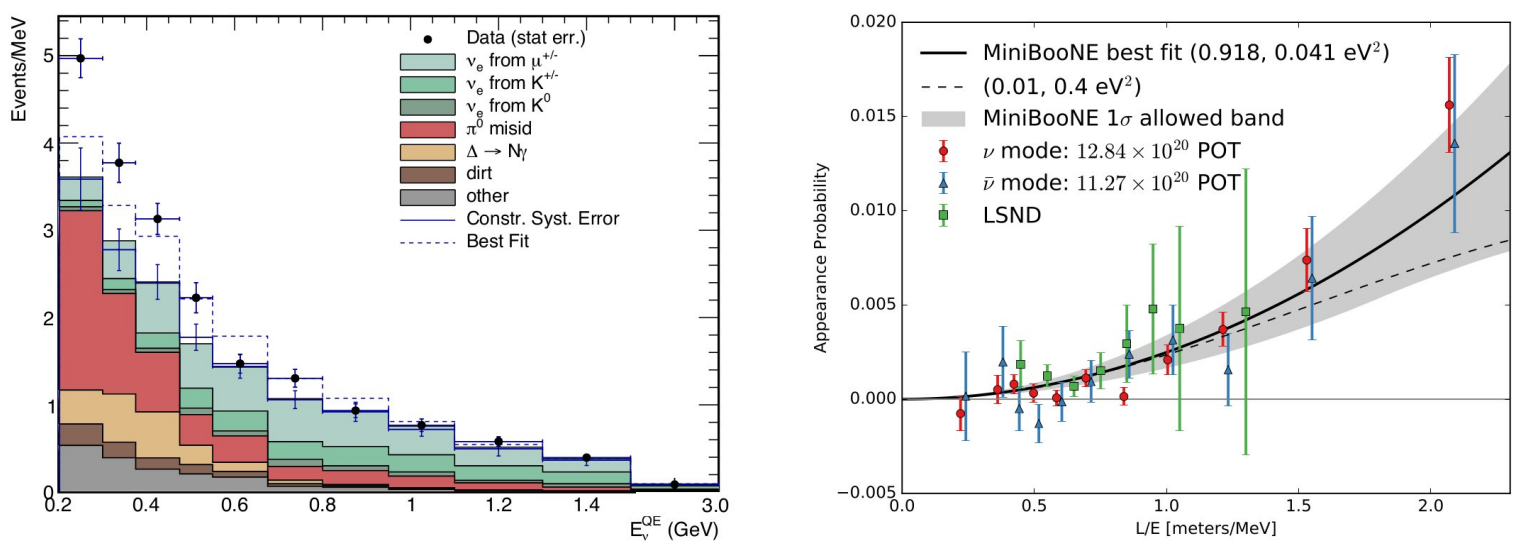

FIGURE 1: (Left) The reconstructed neutrino energy under the charged-current quasielastic interaction hypothesis. The excess in data is visible at low energies. The dotted line corresponds to the best-fit of the data plus background to a sterile neutrino. (Right) The allowed parameter space by the LSND and MiniBooNE results for a sterile neutrino. Figure from [3].

\section{THE MICROBOONE EXPERIMENT}

The MicroBooNE experiment at Fermilab is located within $20 \mathrm{~m}$ of MiniBooNE and employs a LArTPC [4]. MicroBooNE gets neutrinos from two neutrino beams, the Booster Neutrino Beam (BNB) and NuMI (neutrinos from Main Injector). The BNB is the primary neutrino source and on-axis. BNB data has been accumulated by the collaboration over the past 4.5 years, since fall 2015 , and neutrino data equivalent to $\approx 1 \times 10^{21}$ POT has been collected. The flux in neutrino mode has an intrinsic electron neutrino component of $\approx 0.5 \%$, mainly originating from the $\pi^{+} \rightarrow \mu^{+}$channel with subsequent muon decay in flight. The different contributions to the flux are given in fig. 2. 

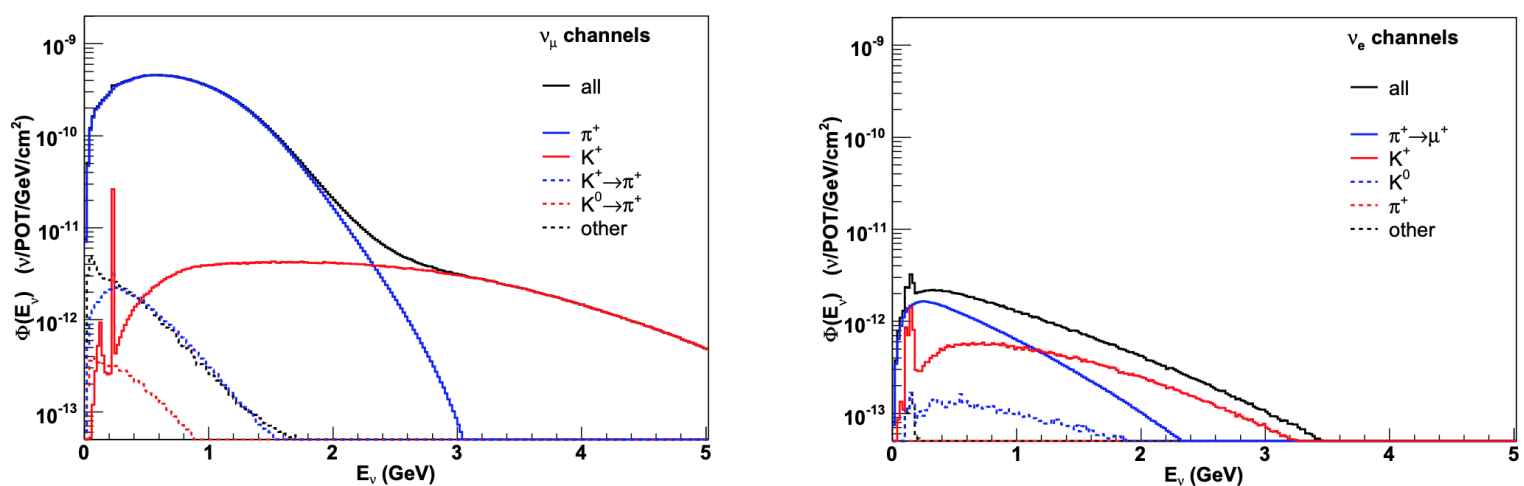

FIGURE 2: Predicted $v_{\mu}$ (left) and $v_{e}$ (right) BNB fluxes at the MicroBooNE detector by parent meson species with horn in neutrino mode. The black line is the total predicted flux. Figure from [5].

The LARTPC technology enables to differentiate between electrons and photons by two characteristics. First, since photons carry no charge, and therefore do not ionise the liquid argon, they only become detectable after undergoing pair-production or Compton scattering. Second, in the case of pair production, the aligned electron-positron pair leads to twice the $\mathrm{d} E / \mathrm{d} x$ an electron at the start of a shower.

\section{THE SEARCH FOR AN ELECTRON NEUTRINO ANOMALY}

The MicroBooNE low-energy excess analysis aims to cover the full physics phase space by looking at both the photon-like excess and the electron neutrino-like excess hypotheses in several channels using the BNB beam. All of these are currently being finalised before unblinding a significant portion of the four and a half year of accumulated BNB data. Therefore, we will look at a set of important side bands and continue by discussing the main sources of systematic uncertainties.

\subsection{Photon Characterisation Using Neutral Pions}

Although the LArTPC technology offers unprecedented particle-identification abilities, the neutrino-induced backgrounds are two orders of magnitude larger. A particularly challenging background category for an electron neutrino selection are neutrino interactions with a $\pi^{0} \rightarrow \gamma \gamma$ in the final state. There are several reasons why such events can be mis-identified as $v_{e}^{\prime}$ s. At first, at the reconstruction stage, one of the two electromagnetic showers might not be reconstructed. this can be because it is especially low in energy $-\mathcal{O}(100 \mathrm{MeV})$ or below - or because both showers are co-linear. Furthermore, for events on the edges of the detector, one of the photons can escape the TPC and go unnoticed.

In cases where one of the two showers is not identified, two features still allow to separate electrons from photons, as illustrated for photons in fig. 3. While in the majority of the cases, the start of the photon shower corresponds to two minimum ionising particles, as created by pair production. In the case of Compton scattering, only a single electron is created, leading to a bump at $2.1 \mathrm{MeV}$. Additionally, the photon conversion distance - measured to be $\approx 28 \mathrm{~cm}-$ follows an exponential distribution. Some photons will interact near the neutrino vertex, masking the gap. The separation is additionally complicated in events without vertex activity (protons, pion) were the vertex distance cannot be used.
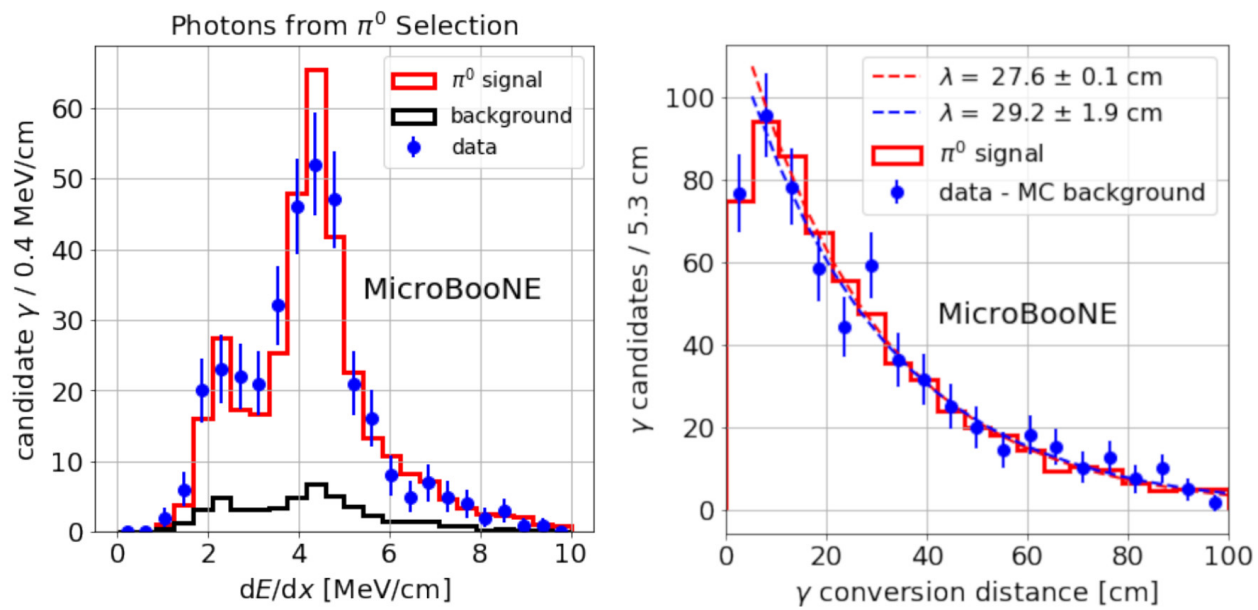

FIGURE 3: (Left) $\mathrm{d} E / \mathrm{d} x$ for photon candidates from selected charged-current muon-neutrino interactions with a $\pi^{0}$ in the final state. (Right) Background subtracted photon conversion distance distributions and fit to an exponential. Figure from [6]. 


\subsection{Electron Neutrino Interactions in the NuMI Beam}

As introduced before, MicroBooNE receives off-axis neutrinos from the NuMI beam. Apart of being unblinded, the intrinsic electron-neutrino beam component is approximately 10 times higher compared to the BNB beam, making the muon-neutrino background rejection less challenging. The result of such a charged-current electron neutrino selection, along with an example data-event is given in fig. 4 . This demonstrates the capability of MicroBooNE to reconstruct and identify electron neutrinos.
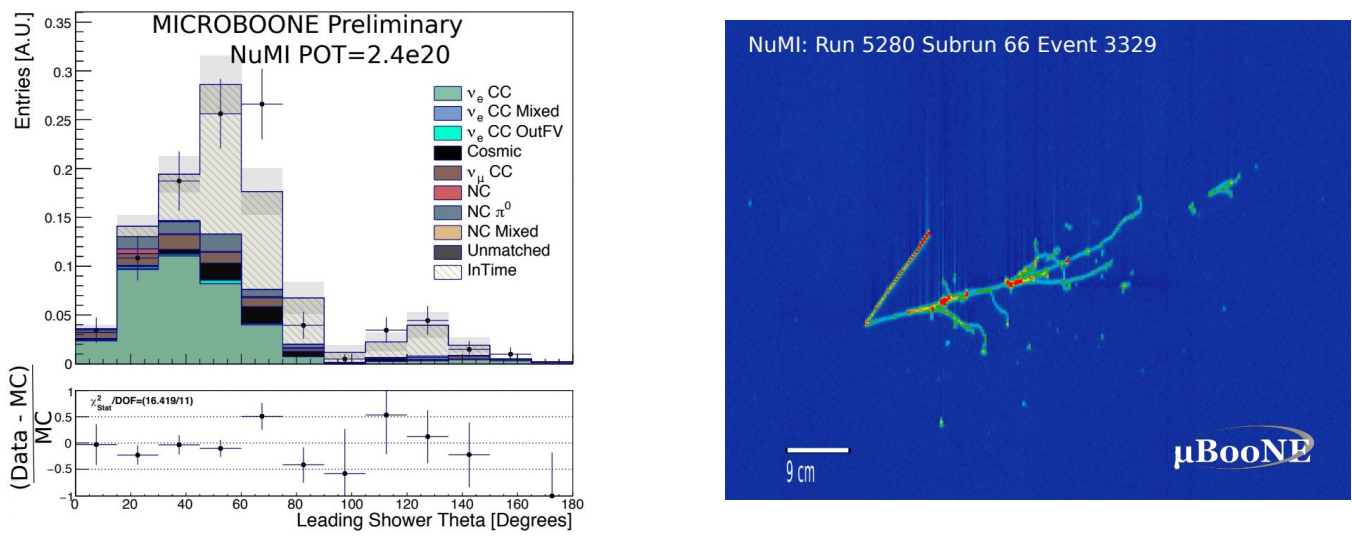

FIGURE 4: (Left) Electron candidate shower angle w.r.t. the beam direction for selected NuMI electron neutrino events. (Right) Event display of an electron neutrino event in NuMI data as observed in MicroBooNE. Figure from [7].

\subsection{Systematic Uncertainties}

The final electron-neutrino selection in MicroBooNE, tailored to the low-energy excess, is expected to select $\mathcal{O}(100)$ events in the data-set. Therefore, the analysis uncertainties are expected to be dominated by statistical fluctuations. The different sources of systematic uncertainties can be grouped into BNB flux, cross-section modelling, and detector variations. The flux variations mainly impact the normalisation and electron neutrino beam content, changing the expected number of neutrino interactions. The crosssection uncertainties affect the contributions of different interaction types and final states. Additionally, cross-section uncertainties impact the reconstructed energy resolution.

To reduce the systematic uncertainties, a data-driven procedure is followed. As was anticipated in fig. 2, the flux of electron neutrinos from the BNB is highly correlated to the muon flux through interlinked production mechanisms. Furthermore, a large set of cross-section uncertainties and detector variations affect the reconstructed muon- and electron-neutrino events similarly. Therefore, a pure sample of contained charged-current muon neutrino interactions is used to constrain the electron neutrino uncertainties. The fractional uncertainties after performing the constraint are approximately a factor two smaller.

\section{CONCLUSION}

It is demonstrated that the MicroBooNE collaboration understands the different steps needed to perform a set of measurements aimed at the investigation of MiniBooNE's low-energy excess. Electromagnetic showers and electron neutrinos were studied with high statistics using charged-current $v_{\mu} \pi^{0}$ events and electron-neutrino interactions in the NuMI beam respectively. MicroBooNE aims to perform a search in several channels, both electron- and photon-like, to clarify the nature of a possible excess. The majority of systematic uncertainties will be constrained using a data-driven method relying on high-statistics muon-neutrino measurements.

\section{References}

[1] A. Diaz, C. A. Argüelles, G. H. Collin, J. M. Conrad and M. H. Shaevitz, arXiv 1906.00045 [hep-ex] (2019)

[2] LSND Collaboration, Phys. Rev. D64, 112007 (2001).

[3] MiniBooNE Collaboration, Phys. Rev. Lett. 121, 221801 (2018).

[4] MicroBooNE Collaboration, JINST 12, P02017 (2017).

[5] MiniBooNE Collaboration, Phys.Rev. D79, 072002 (2008).

[6] MicroBooNE Collaboration, JINST 15, P02007 (2020).

[7] MicroBooNE Collaboration, NOTE-1054-PUB (2018). 